\title{
Revisión de la producción académica latinoamericana sobre desigualdades en mortalidad 2007-2014*
}

\section{A Review of Latin American Academic Production on Inequalities in Mortality 2007-2014}

\section{Revisão de pesquisas acadêmicas latino-americana sobre as desigualdades na mortalidade 2007-2014}

Fecha de recepción: 20-10-2016 Fecha de aceptación: 20-01-2017 Disponible en línea: 30-05-2017 doi:10.11144/Javeriana.rgps16-32.rpal

Cómo citar este artículo:

Rojas-Esguerra DF, Zarama-Tobar IL, Borrero-Ramírez YE, Arrivillaga-Quintero M. Revisión de la producción académica latinoamericana sobre desigualdades en mortalidad 2007-2014. Rev Gerenc Polít Salud. 2017; 16 (32): 10-32. https://doi.org/10.11144/Javeriana.rgps16-32.rpal

\author{
Diego Fernando Rojas-Esguerra** \\ Iris Lamar Zarama-Tobar*** \\ Yadira Eugenia Borrero-Ramírez ${ }^{* * *}$ \\ Marcela Arrivillaga-Quintero*****
}

\footnotetext{
* Artículo de investigación inscrito en el proyecto Inequidades sociales y en mortalidad entre comunas y zona rural de la ciudad de Cali, Colombia, durante el periodo 2000-2011. Fase 1, 15/01/2015 - 30/06/2016, financiado por la Pontificia Universidad Javeriana, Cali, Colombia.

** Magíster en Salud Pública, Pontificia Universidad Javeriana, Cali; docente de la Fundación Universitaria María Cano, Seccional Popayán, y de la Pontificia Universidad Javeriana, Cali. Dirección de correspondencia: Calle 18 No. 118-250 Cali, Colombia. Correo electrónico diegofer1216@hotmail.com

*** Magíster en Salud Pública, Pontificia Universidad Javeriana, Cali; especialista en Fisioterapia Cardiopulmonar, Universidad del Valle; docente de la Fundación Universitaria María Cano, Seccional Popayán. Correo electrónico iriszarama@hotmail.com

**** Ph. D. en Salud Pública, docente e investigadora del grupo Gestión y Políticas de Salud, Facultad Nacional de Salud Pública, Universidad de Antioquia, Medellín, Colombia. Correo electrónico: yadira.borrero@udea.edu.co

***** Psicóloga, magíster en Educación; Ph. D. en Salud Pública; directora del Departamento de Salud Pública y Epidemiología de la Pontificia Universidad Javeriana, Cali; investigadora del Grupo de Investigación de Desarrollo Regional. Correo electrónico.marceq@javerianacali.edu.co
} 


\section{Resumen}

El artículo es una revisión de literatura sobre desigualdades/inequidades en salud en Latinoamérica. Se revisaron Scielo y Lilacs entre 2000 y 2014. Los artículos se clasificaron en tres categorías: mortalidad y artículos teóricos, desigualdades en morbilidad y autopercepción del estado de salud y acceso a servicios de salud. Se realizó análisis crítico de artículos de mortalidad entre 2007 y 2014 , periodo con mayor proporción de producción académica. Se encontraron más investigaciones en Brasil, Colombia y México, especialmente en universidades, con redes de colaboración incipientes. Las metodologías más utilizadas fueron revisiones teóricas, estudios ecológicos y transversales, y predominó el modelo de determinantes sociales de la salud de la OMS. Las categorías empleadas fueron: clase social, posición económica, género, etnia/raza y unidad geográfica. Se concluyó que hay brechas entre la discusión teórica y las investigaciones empíricas, concentración de la producción en pocos países, escaso uso de otros enfoques teóricos y necesidad de fortalecer la colaboración entre instituciones.

Palabras clave: desigualdades en salud; determinantes sociales de la salud; mortalidad; estado de salud

\section{Abstract}

This article is a literature review on inequalities/inequities in health in Latin America. Scielo and Lilacs databases were reviewed between the years 2000 and 2014. The articles were classified into three categories: mortality and theoretical articles, inequalities in morbidity and self-perception of health condition, and access to health services. We carried out a critical analysis of mortality articles between the years 2007 and 2014, the period with the highest proportion of academic production. More research articles were found in Brazil, Colombia, and Mexico, especially in universities with emerging collaboration networks. The most used methodologies were theoretical reviews, ecological and cross-section studies, and a prevailing use of the model of social determinants of health of the WHO. The categories used were: social class, economic position, gender, ethnicity/race, and geographical unit. It was concluded that there are gaps between the theoretical discussion and the empirical research, a concentration of production in a few countries, little use of other theoretical approaches, and a need to strengthen collaboration between institutions.

Keywords: health inequalities; social determinants of health; mortality; health condition

\section{Resumo}

O artigo é revisão de literatura sobre desigualdades/inequidades em saúde na América Latina. Revisaram-se Scielo e Lilacs entre 2000 e 2014. Os artigos foram classificados em três categorias: mortalidade e artigos teóricos, desigualdades em morbidade e autopercepção do estado de saúde e acesso a serviços de saúde. Análise crítica de artigos de mortalidade entre 2007 e 2014, período com maior proporção de produção académica, foi realizada. Encontraram-se mais pesquisas no Brasil, Colômbia e México, especialmente em universidades, com redes de colaboração emergindo. As metodologias mais utilizadas foram revisões teóricas, estudos ecológicos e transversais, e predominou o modelo de determinantes sociais da saúde da OMS. As categorias empregadas foram: camada social, posição económica, género, etnia/raça e unidade geográfica. Concluiu-se que tem fendas entre discussão teórica e pesquisas empíricas, concentração da produção em poucos países, utilização insuficiente de outros enfoques teóricos e necessidade de fortalecer a colaboração entre instituições.

Palavras chave: desigualdades em saúde; determinantes sociais da saúde; mortalidade; estado de saúde. 


\section{Introducción}

$\mathrm{El}$ aumento de las desigualdades/inequidades sociales en salud durante las últimas décadas ha coincidido con procesos de reforma del Estado de tipo económico, de política social y de sistemas de salud y seguridad social, en distintos países y con una fuerte orientación hacia el mercado (1-3). Estas transformaciones se implementaron con el argumento de solucionar la crisis de la deuda experimentada por los países en vía de desarrollo en la década de los años ochenta (3). En la formulación de estas políticas —de ajuste estructural- desempeñaron un papel clave el Banco Mundial (BM) y el Fondo Monetario Internacional (FMI), quienes orientaron a países endeudados hacia la adopción de reformas como condición para conceder nuevos créditos o disminuir las tasas de interés sobre estos (13). La implementación de dichas políticas, llamadas reformas neoliberales, consistió en disminuir el protagonismo del Estado en la formulación e implementación de políticas sociales y trasladar a los actores privados la responsabilidad de lo social, así como convertir la implementación de tales políticas en un nuevo escenario para la acumulación de capital (3).

En Latinoamérica se han sentido los efectos de estas reformas. Si bien, inicialmente, se pensó de manera optimista que los beneficios acumulados por los operadores privados y la estabilización económica tendrían un efecto de filtrado o "derrame", que en el mediano plazo favorecería el aumento de los ingresos de la mayoría de la población, esto no sucedió y, por el contrario, aumentó la pobreza y el desempleo, lo que se pensó serían efectos momentáneos (3). Este optimismo inicial se perdió cuando a mediados de los noventa los países de la región enfrentaron una nueva crisis de la deuda, con una subsecuente recesión económica -México 1994-1995, Colombia 1998-1999, Brasil 1999 y Argentina 20012002- (3). Esos años también coincidieron con un aumento sin precedentes de los beneficios del sector privado (3). Por lo anterior, no es de sorprender que Latinoamérica sea una de las regiones con mayor desigualdad en el mundo. En la región existe heterogeneidad en resultados relacionados con la riqueza: el quintil más pobre recibe un $5 \%$ del ingreso total, variando desde menos del $4 \%$ en Honduras, Paraguay y República Dominicana hasta $10 \%$ en Uruguay, mientras que el quintil más rico posee el $47 \%$, con un rango entre $35 \%$ en Uruguay y $55 \%$ en Brasil (4).

Respecto a los altos niveles de desigualdad registrados en la región, estos tienen sus causas fundamentales en desigualdades históricas en aspectos como: posesión de tierra, discriminación étnica, baja recaudación de impuestos, sumado a los efectos nocivos de las reformas neoliberales aplicadas (5). Pese a esto, en los últimos años hay desarrollos importantes, impulsados por gobiernos alternativos, como en el caso de Argentina, Bolivia, Brasil, Chile, Ecuador, Paraguay, Uruguay y Venezuela, desafiando nociones hegemónicas del Consenso de Washington de que no hay alternativa a la reducción de la intervención y la desregulación de los mercados laborales, financieros y comerciales (2).

Este panorama de desigualdades ha impactado de manera importante los resultados de salud, diversos estudios coinciden en que el nivel de salud de grupos en mejor posición socioeconómica y que viven en sectores privilegiados es mucho mejor que el de la población menos favorecida (6); por consiguiente, las diferencias en salud entre distintos grupos según posición socioeconómica y área geográfica, entre otros factores, configura las denominadas desigualdades/inequidades sociales en salud.

En relación con ello, en los últimos años existe un fuerte interés, por parte de instituciones e investigadores en salud pública de Latinoamérica, en la investigación de las 
desigualdades/inequidades en salud, en un contexto en el que las diferencias socioeconómicas son muy marcadas, lo cual se evidencia en el incremento de la producción académica al respecto (7). Dentro de dicha producción se encontraron revisiones sistemáticas de Almeida-Filho (8,9), análisis bibliométrico de Raiher (10), revisión sistemática sobre producción académica en Brasil de Labernarde y Engelhardt (11), el mapeo de capacidades para la investigación sobre determinantes de la salud en Brasil de Borde et al. (12) y la revisión de clase social de Muntaner et al. (13).

Pese a los anteriores trabajos, no se encontraron investigaciones que analizaran la producción académica en el campo de las desigualdades/inequidades en mortalidad en Latinoamérica, que dieran cuenta de perspectivas teóricas, categorías de análisis, países e instituciones donde se investiga y redes de colaboración. Por tal motivo, el presente trabajo responde a la pregunta: ¿Cómo ha sido la producción académica en Latinoamérica sobre desigualdades sociales en salud, para el periodo comprendido entre los años 2000 y 2014? El objetivo fue analizar la producción académica sobre el tema de inequidades/desigualdades en mortalidad en la región, identificando: perspectivas teóricas, categorías de análisis, metodologías de investigación, así como centros producción académica y redes de colaboración de instituciones de la región.

\section{Método}

Inicialmente, este estudio correspondió a una revisión temática de la producción académica en Latinoamérica sobre desigualdades sociales en salud, en el periodo 2000-2014. Para tal fin, se realizó una búsqueda de artículos publicados en las bases de datos de Scielo y Lilacs, las cuales fueron seleccionadas porque incluyen la producción académica de la región y con artículos de libre acceso. La búsqueda se realizó con los descriptores: desigualdades en salud, equidad en salud e inequidades en salud, en los idiomas español, inglés y portugués. Los criterios de inclusión fueron: artículos publicados durante el periodo de investigación sobre temáticas de desigualdades/inequidades sociales en salud en Latinoamérica, en las bases de datos definidas, con las palabras clave mencionadas y publicados por autores latinoamericanos, desde Latinoamérica y sobre la región. Se excluyeron: editoriales, artículos de opinión, documentos oficiales y no gubernamentales, así como artículos sobre Latinoamérica sin autores latinoamericanos.

La información inicial recopilada sobre los artículos fue: año de publicación, palabra clave, nombre, autor y tipo de desigualdad abordada. Dicha búsqueda arrojó, inicialmente, un total de 1137 artículos (561 en Lilacs y 576 en la base de datos Scielo). Fueron descartados 174 por duplicidad y 79 que no cumplieron los criterios de inclusión.

Los 884 artículos fueron clasificados en tres grupos: artículos sobre desigualdades en mortalidad y teóricos — se incluyeron los teóricos dado que en las investigaciones empíricas no fue usual encontrar discusiones teóricas sobre el tema-, con un total de 202 artículos; artículos sobre desigualdades en patologías específicas y autopercepción del estado de salud, con 435 artículos, y artículos sobre desigualdades en el acceso a los servicios de salud, con 247 artículos.

Posteriormente, y dado el volumen de la producción académica y los objetivos del trabajo, se seleccionaron los estudios teóricos y de mortalidad entre el 2007 y el 2014. Este último año se seleccionó porque en él se encontró un incremento importante de la producción académica. Se consideró: lugar de producción, redes de colaboración, revistas de publicación, perspectivas teóricas, diseño metodológico, categorías e indicadores usados 
para el análisis y resultados. Finalmente, se incluyeron cien artículos.

\section{Resultados}

Volumen y tendencia de la producción académica 2000-2014

Se encontró que la producción sobre desigualdades/inequidades en salud en la región, para el periodo, ha sido importante; en total hubo 884 artículos publicados en revistas indexadas, cuyos temas correspondían a desigualdades en mortalidad, morbilidad, autopercepción del estado de salud, acceso a servicios de salud, y debates teórico-metodológicos. A partir del
2007 hubo un incremento de la producción académica (figura 1). El $23 \%$ de los estudios estuvo dentro del grupo 1 -mortalidad y discusión teórico-metodológica-; el $49 \%$ correspondió a estudios sobre desigualdades en morbilidad por patologías específicas y autopercepción del estado de salud, y el $28 \%$ a estudios sobre desigualdad en el acceso a los servicios de salud. La tendencia a lo largo del periodo ha sido de incremento sostenido de la producción académica. Seguramente, el aumento observado a partir del 2007 se relaciona con los desarrollos liderados desde la Organización Mundial de la Salud (OMS) en torno al debate sobre desigualdades en salud de la Comisión de Determinantes Sociales de la Salud (CDSS).

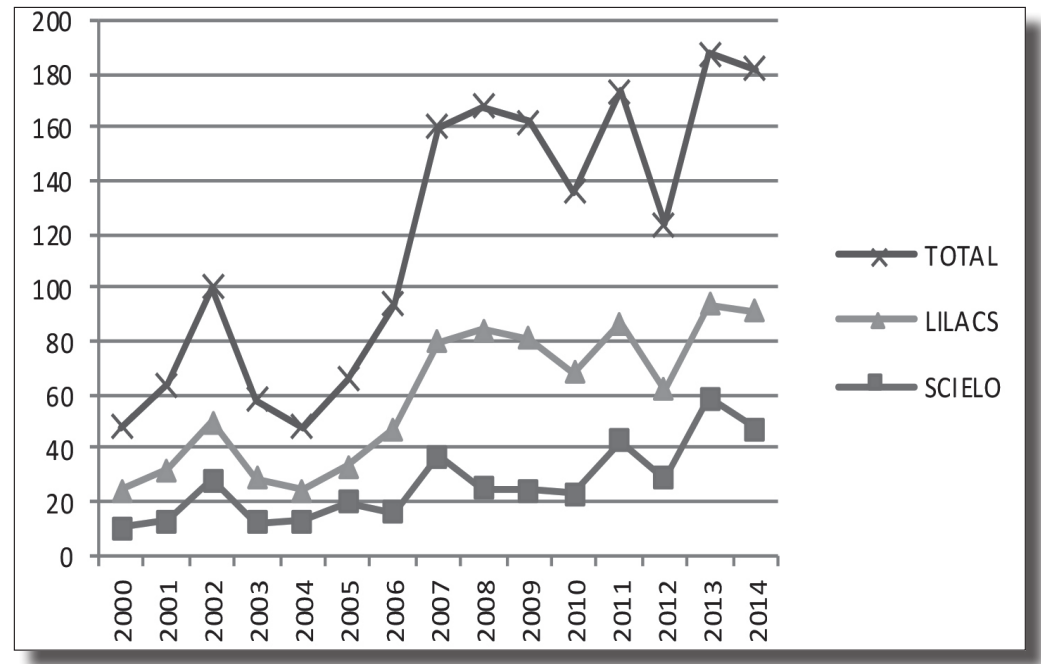

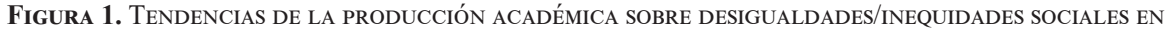
SALUD EN LATINOAMÉRICA 2000-2014

Fuente: elaboración propia

Producción académica 2007- 2014: países, centros y redes

Países, centros y publicaciones donde se genera la investigación sobre desigualda14 des en mortalidad en Latinoamérica. Se encontró que el país con mayor producción académica sobre el tema fue Brasil, con un $47 \%$ del total para el periodo, seguido de Colombia con $20 \%$, México $12 \%$, Cuba $7 \%$, Argentina 5\%, Chile 4\%, Perú $2 \%$ y, con una muy baja representación, Venezuela, Puerto Rico y Costa Rica con $1 \%$ (figura 2). 


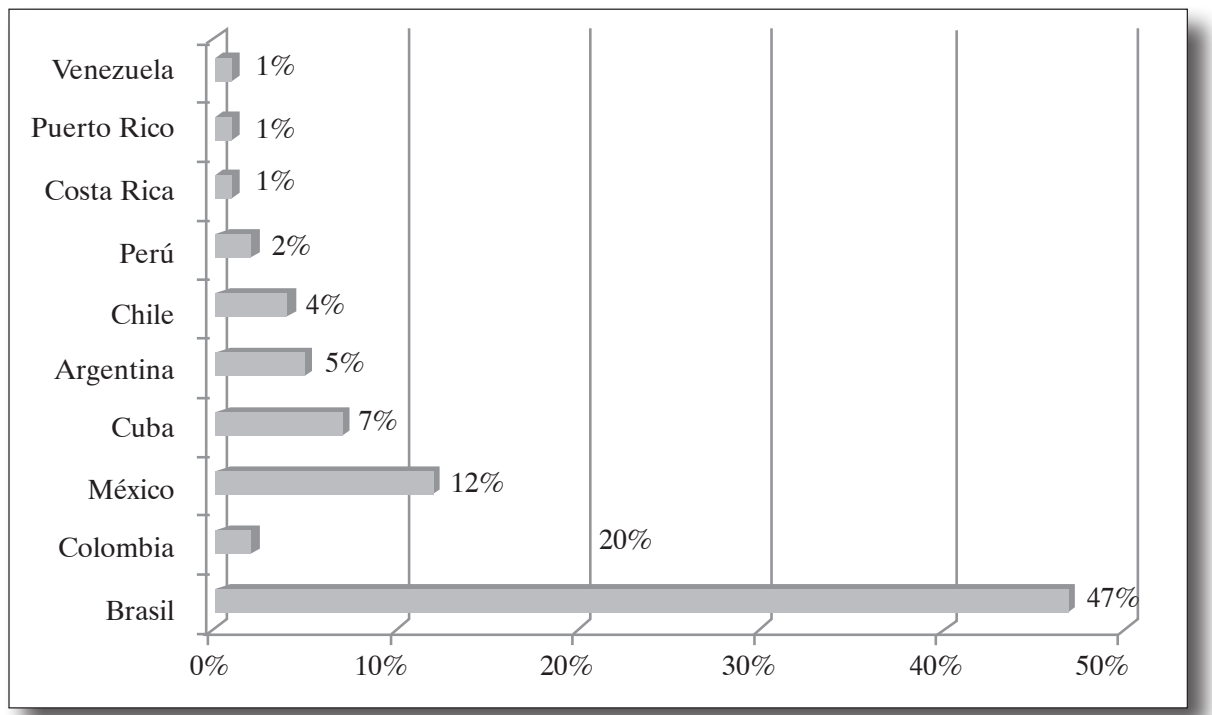

Figura 2. Volumen de Produccion aCADÉmiCA SOBRE DESIGUALDADES POR PAises en AMÉrica Latina. 2007-2014

Fuente: elaboración propia

Además, hay una enorme heterogeneidad en los centros de pensamiento en los cuales hay producción. En Brasil se identificaron treinta instituciones públicas y privadas. Las más importantes, de acuerdo al número de publicaciones, son: Fundación Oswaldo Cruz (seis), Universidad Federal Do Rio Grande Do Soul (cuatro), Universidad Federal de Bahía, Universidad de Sao Paulo (tres), Universidad Estadual de Campinas (dos). Colombia ocupa el segundo lugar en producción académica, los centros con mayor producción fueron: Universidad Nacional de Colombia (seis), Universidad de Antioquia (cinco) y la Pontificia Universidad Javeriana (cuatro). México aporta producción en el área de estudio; ocupa el tercer lugar, los centros son Instituto Nacional de Salud Pública de México (tres), Universidad de Guadalajara e Instituto Nacional de Geriatría. La producción académica se genera desde ámbitos universitarios por excelencia.
Las revistas que publican están principalmente en Brasil, Colombia y Cuba. En las revistas se ratifica la importancia de las mismas instituciones académicas, con Cadernos de Saude Publica (Fiocruz) en primer lugar, seguida de la Revista de la Facultad Nacional de Salud Pública (Universidad de Antioquia), Revista de Salud Pública (Universidad Nacional de Colombia), Ciencia y Saude Coletiva (Asociación Brasilera de Salud Colectiva Abrasco), Revista Panamericana de Salud Pública (Organización Panamericana de la Salud [OPS]), Revista de Saude Pública (Universidad de Sao Paulo), Revista Cubana de Salud Pública (Centro Nacional de información en Ciencias Médicas) y Revista Brasilera de Epidemiologia (Asociación Brasilera de Salud Colectiva [Abrasco]). Lo anterior ratifica la importancia de Brasil, no solo en la producción académica, sino en su difusión. Fiocruz y Abrasco se constituyen como instituciones claves para la publicación de trabajos sobre el tema en la región. 
Redes de colaboración. Sobre las redes de colaboración se encontró que casi la mitad de las identificadas son entre instituciones brasileras. En general, la colaboración entre instituciones de los diferentes países es poca. En este sentido, las redes identificadas en Latinoamérica fueron cuatro: Universidad CES (Colombia)-Universidad Nacional de Córdoba (Argentina); Uni- versidad Nacional de Colombia-Fiocruz (Brasil)-Universidad Santo Tomas de Bucaramanga (Colombia); Universidad Nacional de Lanús (Argentina)-Universidad Federal de Bahía (Brasil); Instituto Nacional de Salud Pública (México)-Universidad Industrial de Santander (Colombia)-Universidad Nacional Autónoma de México (figura 3).

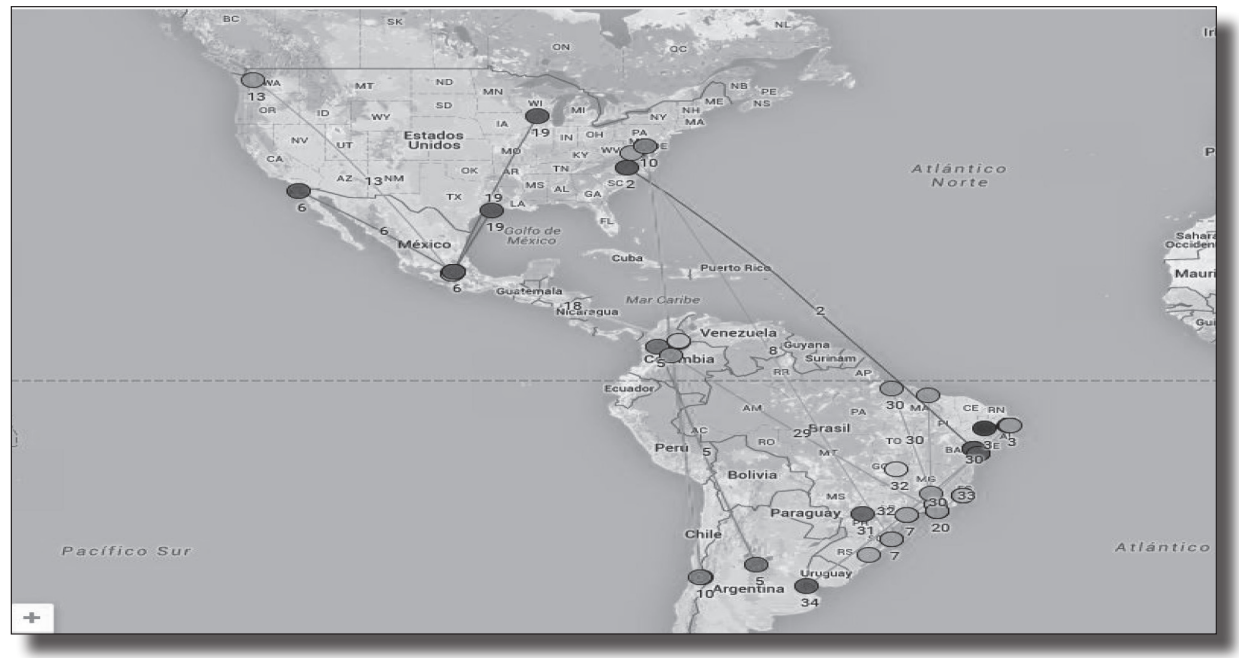

Figura 3. Redes de COlaboración entre países de AmÉrica

Fuente: elaboración propia

Asimismo, se identificaron cinco redes de colaboración entre instituciones latinoamericanas y de Estados Unidos. Las instituciones mexicanas y brasileñas son las que más han trabajado con universidades estadounidenses: Instituto Nacional de Salud Pública-Universidad de Washington; Instituto Nacional de Geriatría de México-Universidad de Texas-Universidad de Wisconsin; Universidad de Feira de Santana-Universidad de Carolina del Norte; Universidad Federal de Santa Catarina-Universidad de Virginia; Universidad del Desarrollo (Chile)16 Organización Panamericana de la Salud, Washington (figura 3).
Además, se encontró una red de colaboración con instituciones europeas: Instituto Nacional de Higiene, Epidemiología y Microbiología (Cuba)-Instituto de Medicina Tropical de Amberes (Bélgica), y una red en la que participan instituciones de Norteamérica, Suramérica y Europa, conformada por la Universidad CES (Colombia)Erasmus University Medical Center (Países Bajos)-London School of Economics and Political Science (Reino Unido)-Universidad de Harvard (Estados Unidos) (figura 4). Una red con gran importancia en Suramérica es la de la Universidad Nacional de Lanús y la Universidad Federal de Bahía (figura 3). 


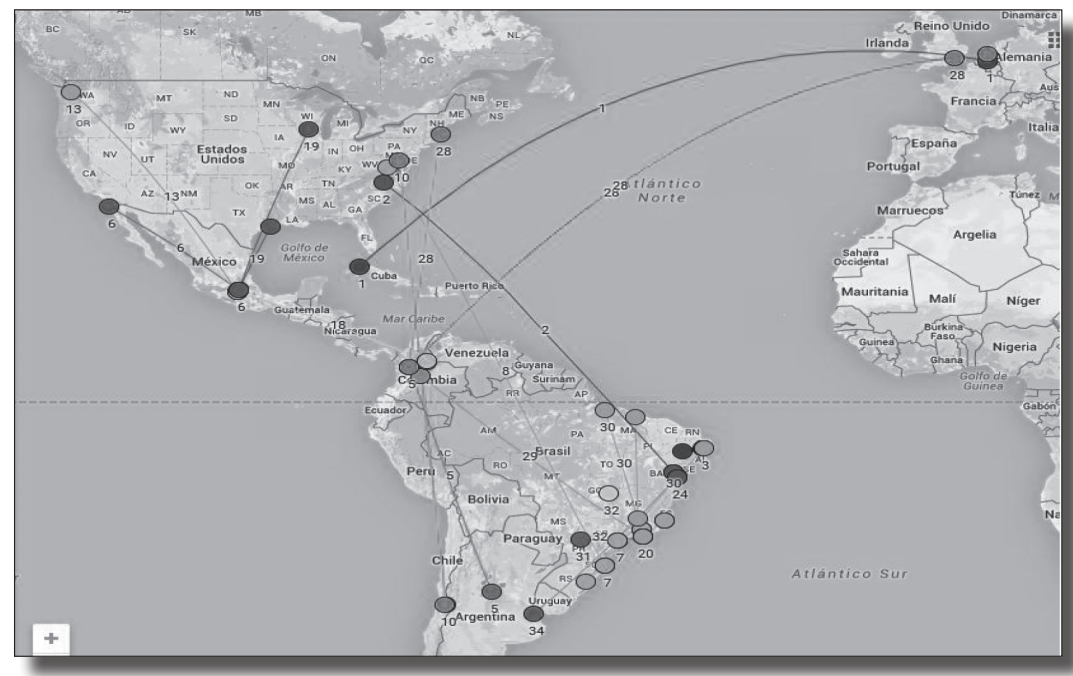

Figura 4. Redes de colaboración con Europa y Norteamérica

Fuente: elaboración propia

\section{Temas de investigación 2007-2014}

Como muestra la tabla 1, en la región se ha dado importancia a la discusión teórico-metodológica, seguida de problemas persistentes como la mortalidad materno-infantil y por enfermedades crónicas, asuntos importantes de la investigación en América Latina.

TABLa 1. DistribuCión DE LOS TEMAS DE INVESTIGACIÓN ENTRE 2007 Y 2014. MORTALIDAD Y DISCUSIONES TEÓRICO-METODOLÓGICAS

\begin{tabular}{|c|c|c|}
\hline Tema & Frecuencia & Porcentaje \\
\hline Debates teóricos/metodológicos & 39 & $39 \%$ \\
\hline Mortalidad materno-infantil & 28 & $28 \%$ \\
\hline Mortalidad por enfermedades crónicas & 11 & $11 \%$ \\
\hline Mortalidad general & 7 & $7 \%$ \\
\hline Otras causas de mortalidad & 6 & $6 \%$ \\
\hline Mortalidad evitable & 4 & $4 \%$ \\
\hline Esperanza de vida al nacer & 5 & $5 \%$ \\
\hline Total & 100 & $100 \%$ \\
\hline
\end{tabular}

Fuente: elaboración propia

\section{Diseños de investigación}

El estudio de las desigualdades/inequidades sociales en salud en Latinoamérica ha privilegiado el uso de métodos epidemiológicos, con énfasis en estudios transversales y ecológicos para abordar las desigualdades en mortalidad. Los estudios cualitativos son absolutamente marginales en la investigación sobre el tema en la región (véase tabla 2). 
TABLA 2. DistribUCIÓN DE LA PRODUCCIÓN ACADÉMICA SOBRE DESIGUALDADES/INEQUIDADES EN MORTALIDAD Y ESTUDIOS TEÓRICOS, SEGÚN DISEÑO METODOLÓGICO

\begin{tabular}{|c|c|c|}
\hline $\begin{array}{c}\text { Diseños de } \\
\text { investigación }\end{array}$ & Frecuencia & Porcentaje \\
\hline Teóricos / debates & 39 & $39 \%$ \\
\hline Ecológicos & 33 & $33 \%$ \\
\hline Transversales & 20 & $20 \%$ \\
\hline Revisión sistemática & 4 & $4 \%$ \\
\hline Cohorte & 3 & $3 \%$ \\
\hline Caso control & 1 & $1 \%$ \\
\hline Total & 100 & $100 \%$ \\
\hline
\end{tabular}

Fuente: elaboración propia

\section{Perspectivas teóricas y categorías de análisis}

Modelos teóricos: determinantes y determinación social de la salud. Dentro de las investigaciones, la mayoría de los trabajos hace alusión explícita a la perspectiva de los determinantes sociales de la salud (DSS), desde el enfoque de la OMS (7,14-38). Marchiori y Pellegrini Filho (30) se basan en dos modelos de determinantes sociales: el modelo de Dahlgren y Whitehead, el cual también es adoptado por Geib (33) y divide los determinantes según niveles, con determinantes proximales y distales, a partir de las características individuales de los individuos, los estilos de vida, seguido de un nuevo nivel donde se ubica la influencia de redes sociales y comunitarias expresadas en la cohesión social, luego el acceso a los servicios de salud, las condiciones de vida y trabajo, hasta llegar a los macrodeterminantes, reflejados en las condiciones económicas, culturales y ambientales. El segundo modelo es el de Diderichsen y Hallqvist, modificado por Diderichsen, Evans y Whitehead, en el que se plantea una relación entre los contextos sociales y la estratificación social, lo cual define la posición social de los individuos, de acuerdo a la educación y el mercado de trabajo; es la posición social la que genera exposición a riesgos diferenciales para la salud en los diferentes grupos sociales.

Cabieses (38), por su parte, acoge la definición de determinantes como las condiciones sociales en que viven y trabajan las personas y que afectan su salud, y adopta el modelo de la Comisión de Determinantes Sociales de la Salud de la OMS (CDSS/OMS). Destaca que la importancia de este modelo radica en que, no solo describe los principales determinantes de acuerdo a la evidencia internacional disponible, sino que además actualiza las relaciones entre dichos componentes y sus efectos directos e indirectos en la salud. Este modelo se organiza en tres niveles: el nivel macro/contextual, el nivel individual, ambos definidos como estructurales, y el nivel intermediario en salud, que representa el nivel explicativo de desigualdades en salud. Los determinantes intermediarios se comportan como categorías de mediación entre la estructura y la familia y los individuos, aspectos como circunstancias en que se vive, factores psicosociales y factores conductuales y biológicos. La posición social, el género y la etnia son determinantes planteados en el nivel estructural junto con las políticas públicas.

De otro lado, algunos estudios de tipo teórico asumen como referente el modelo de determinación social (39-43). Este modelo 
discute las aproximaciones de los determinantes de la salud impulsadas por la CDSS, a partir de varias críticas, entre ellas: de un lado, la división entre determinantes proximales y distales, que termina manejando los determinantes proximales como factores de riesgo, y, de otro lado, el menor énfasis en la discusión de los determinantes estructurales como producto del modelo de desarrollo capitalista que genera explotación extrema de los recursos naturales y los seres humanos, en función de los procesos de acumulación de capital.

Los hallazgos muestran que las instituciones latinoamericanas y sus investigadores asumen principalmente el modelo de determinantes de la salud de la OMS, pese al debate impulsado desde los años setenta por la medicina social latinoamericana, la epidemiología crítica y la salud colectiva. Esto es aún más evidente para las investigaciones empíricas, las cuales operacionalizan en su mayoría el modelo OMS y privilegian — como se verá más adelante- análisis de la posición social y la pertenencia de género o étnica. Respecto a la salud colectiva, es importante el estudio de Borde et al. (12), quienes plantean que pese al liderazgo de Brasil en la investigación sobre determinantes y de las acciones desde la salud colectiva brasileña, aún falta mayor difusión e impacto real de la investigación, probablemente porque la mayor parte de los artículos son escritos en portugués, la escritura en inglés es muy reducida y los trabajos en red internacional de las instituciones brasileñas son escasos.

\section{Categorías explicativas de las desigualdades/} inequidades usadas en la investigación. Se encontraron cinco categorías emergentes explicativas para el análisis de los determinantes de las desigualdades/inequidades en salud, ellas son: condiciones socioeconómicas de individuos y familias, género, etnia o raza, condiciones socioeconómicas de unidades territoriales y factores de riesgo. Estas se revisan a continuación.

Clase social, posición socioeconómica: una de las categorías más usadas para analizar y explicar las desigualdades en salud son las condiciones socioeconómicas de grupos sociales o individuos. De hecho, este análisis fue el más frecuente, así como el más discutido teórica y metodológicamente. Dentro de la revisión se encontraron tres tipos de abordajes: clase social, posición socioeconómica y perspectiva de capacidades.

La categoría clase social construye una mirada relacional, de tipo conflictivo, entre grupos sociales con diferentes posiciones respecto a los medios de producción. Se ha trabajado desde dos enfoques: perspectivas neomarxistas, que beben de la discusión marxista de división en clases sociales en el capitalismo, según tenencia de los medios de producción, y la tradición weberiana del siglo XIX y de Bourdieu en el XX, que suma a lo anterior otros elementos constitutivos como prácticas y costumbres, reconocimiento, estatus y participación en el mercado laboral. La clase social de Wright $(19,44)$ clasifica los grupos según la relación con la propiedad, el control sobre la organización del trabajo y credenciales/habilidades. Básicamente, esta clasificación divide la clase social en propietarios y asalariados. De otro lado, la perspectiva de Goldthorpe, desde un enfoque neoweberiano, privilegia para la clasificación, además de la tenencia de los medios de producción, las credenciales y habilidades como recurso social y de distinción. La concepción más usada en la región es neomarxista, coincidiendo con los hallazgos de Muntaner et al. (13), quienes en la revisión sobre la producción académica de DSS y clase social encontraron que existe una carencia de estudios que aborden el tema de los DSS en el ámbito latinoamericano; sin embargo, en dicha revisión se encontró que 
la mayoría utilizó la teoría neomarxista para la definición de clase social, y estos trabajos se realizaron principalmente en Brasil.

La segunda perspectiva encontrada fue la posición socioeconómica, para la cual se usó información, básicamente, de nivel educativo, ocupación e ingreso; no incluye información sobre la relación con los medios de producción (véase tabla 3). La última perspectiva encontrada fue con base en el paradigma de las capacidades, que se fundamenta en los principios de libertad y oportunidad. La libertad, vinculada con la realización de las potencialidades individuales que permitan conseguir las metas para tener acceso a bienes y servicios que mejoren el estado de salud, y la capacidad adquisitiva, para poder pagar bienes de salud como medicamentos o consultas médicas especializadas. Lo anterior se refuerza con el capital social, para dar solución a dificultades experimentadas por los individuos. De esta manera, se brindan las oportunidades para que cada persona logre mejorar su situación en salud (45).

Tabla 3. Medidas de Posición SocioeconómiCa y ClaSe usadas dentro de las inVestigaciones SOBRE MORTALIDAD 2007-2014

\begin{tabular}{|l|l|c|}
\hline \multicolumn{1}{|c|}{ Tipo de indicador } & \multicolumn{1}{|c|}{ Definición } & $\begin{array}{c}\text { Investigaciones } \\
\text { que lo usan }\end{array}$ \\
\hline $\begin{array}{l}\text { Coeficiente Gini en } \\
\text { la distribución de } \\
\text { ingresos }\end{array}$ & $\begin{array}{l}\text { Indicador que expresa } \\
\text { la desigualdad en la } \\
\text { distribución de renta }\end{array}$ & $(46-48)$ \\
\hline Trabajo e ingresos & $\begin{array}{l}\text { Número de personas } \\
\text { del hogar que están } \\
\text { trabajando, condi- } \\
\text { ciones de empleo, } \\
\text { ingresos en salarios } \\
\text { mínimos, acceso a } \\
\text { seguro médico privado }\end{array}$ & \\
\hline $\begin{array}{l}\text { Posición } \\
\text { socioeconómica }\end{array}$ & $\begin{array}{l}\text { Educación e ingresos } \\
\text { familiares de la madre, } \\
\text { el padre o el cuidador }\end{array}$ & \\
\hline
\end{tabular}

Fuente: elaboración propia

Género: siete investigaciones estudiaron desigualdades/inequidades de género (34,41,51-55). En general, pareciera haber un consenso sobre esta categoría, al entender el género como una construcción sociohistórica, mediada por relaciones de poder entre hombres y mujeres, donde las mujeres ocupan un lugar subordinado en la sociedad latinoamericana; sin embargo, no se trabajan mucho ni identidades de género ni orientaciones sexuales no heteronormativas, siendo esto una limitación de los estudios de género en la región.
De particular interés resulta el estudio de Artiles (51), en el que se analizan las principales causas de las desigualdades/inequidades en salud que afectan a las mujeres: la esperanza de vida, que es mayor en las mujeres, puede prestarse a errores en su comprensión, puesto que en las comunidades más pobres las mujeres mueren de manera más prematura, o su sobrevida es a expensas de malas condiciones de salud. Un segundo aspecto corresponde al ingreso, en cuanto que en todos los países las mujeres tienen un ingreso per cápita menor que los 
hombres, sumado a problemáticas como falta de acceso a la tierra, al crédito, a la educación, falta de autonomía, imposibilidad de planificación familiar, sometimiento y violencia doméstica, entre otros temas. Un tercer aspecto es el nivel educativo. Si bien en la mayoría de los países las mujeres tienen mayor acceso a los servicios educativos, en los más pobres la tendencia es distinta, y esto dificulta el alcance de mayores posibilidades de desarrollo y calidad de vida de la mujer. La participación política es la cuarta dimensión. Pese a que las mujeres han logrado mayor alcance en la ocupación de posiciones de autoridad o toma de decisiones, sigue existiendo una mayoría masculina en estos espacios. Finalmente, una última característica del mundo femenino en el campo de la salud son las particularidades biológicas del cuerpo femenino, relacionadas con rasgos diferentes en cuanto a su salud sexual, reproductiva y a la mayor carga que impone el rol de la maternidad y el papel de proveedoras de cuidado en los hogares (45).

Etnia/raza: en esta categoría todos los trabajos encontrados fueron realizados en Brasil, país caracterizado por su diversidad cultural y étnica, dada la alta proporción de población afro e indígena. Fernández Aneiros (18) hace un recuento histórico de los diferentes paradigmas respecto al proceso salud-enfermedad y destaca que su determinación debe tener en cuenta la cosmovisión de los diferentes grupos étnicos para la formulación de políticas públicas en salud, al igual que para los servicios de salud, de manera que logren adoptar modelos de atención con enfoque intercultural. Resalta que, probablemente, la falta de visión intercultural haya sido la causa de las desigualdades/inequidades en salud de origen étnico/racial. Es decir, ubica una dimensión sociosimbólica para pensar las desigualdades/inequidades en salud entre grupos con diferentes pertenencias étnicas, además de las propias condiciones socioeconómicas. Lo anterior concuerda con los postulados de Figueiredo (19), quien menciona que las desventajas en salud de los grupos étnicos minoritarios obedecen más a aspectos históricos, culturales, de segregación o discriminación que a las desigualdades socioeconómicas, de clase o posición social, y resalta que, incluso en las clases privilegiadas, los afrodescendientes reportan peor percepción de salud respecto a las comunidades blancas. Esta falta de comprensión acerca de las desigualdades/ inequidades en salud de origen étnico se refleja en la revisión realizada por de Araujo et al. (20), quienes encuentran que, pese a que existe una amplia investigación sobre la salud de las minorías étnicas en el ámbito internacional, principalmente en Inglaterra y Estados Unidos, esta investigación aun es incipiente en Brasil, y podemos decir en el resto de Latinoamérica.

\section{Situación socioeconómica de unidades geo-}

gráficas: se encontró que veintisiete estudios trabajaron desigualdades entre unidades geográficas y para ello han usado diferentes indicadores e índices que intentan diferenciar las condiciones socioeconómicas de los territorios. En la tabla 4 se muestran aquellos usados en la investigación a escala regional. Pese al volumen de trabajos encontrados, desafortunadamente, hay ausencia de reflexión teórica en torno a cómo se puede explicar la relación entre las condiciones socioeconómicas del territorio y los resultados en salud. En general, asumen que las poblaciones ubicadas en zonas más distantes o en áreas más pobres son más vulnerables en términos de salud, sin explicar este vínculo. Trabajar conceptualmente la relación entre territorio y salud es un pendiente de la reflexión sobre desigualdades/ inequidades en salud en Latinoamérica. 
TABLA 4. ÍNDICES USADOS PARA MEDICIÓN DE CONDICIONES SOCIOECONÓMICAS EN UNIDADES TERRITORIALES DENTRO DE LAS INVESTIGACIONES SOBRE MORTALIDAD 2007-2014

\begin{tabular}{|c|c|c|c|}
\hline Índices & Componentes & $\begin{array}{c}\text { Autores que lo } \\
\text { usan }\end{array}$ & Observaciones \\
\hline $\begin{array}{l}\text { Índice de } \\
\text { desarrollo } \\
\text { humano }\end{array}$ & $\begin{array}{l}\text { Índice está compuesto de la esperanza de } \\
\text { vida al nacer, la educación y la renta }\end{array}$ & $\begin{array}{c}(46),(56),(57), \\
(15),(17),(27), \\
(48)\end{array}$ & $\begin{array}{l}\text { Se usa en diferentes } \\
\text { unidades territoriales, a } \\
\text { nivel cantón, de munici- } \\
\text { pio, estado, país }\end{array}$ \\
\hline $\begin{array}{l}\text { Condicio- } \\
\text { nes de vida }\end{array}$ & $\begin{array}{l}\text { Tasa de analfabetismo en mayores de cin- } \\
\text { co años, porcentaje de jefes de familia con } \\
\text { menos de cuatro años de escolaridad y } \\
\text { porcentaje de jefes de familia con más de } \\
\text { diez años de escolaridad, renta (medida } \\
\text { en salarios mínimos) }\end{array}$ & $\begin{array}{l}(58),(54),(57), \\
\quad(26),(59)\end{array}$ & $\begin{array}{l}\text { Este índice ha sido usa- } \\
\text { do por varios estudios, } \\
\text { algunos incluyen otras } \\
\text { variables como propor- } \\
\text { ción de domicilios con } \\
\text { agua potable, propor- } \\
\text { ción de domicilios con } \\
\text { instalaciones sanitarias, } \\
\text { proporción de pobla- } \\
\text { ción de diez a catorce } \\
\text { años analfabeta }\end{array}$ \\
\hline $\begin{array}{l}\text { Índice de } \\
\text { desarro- } \\
\text { llo de la } \\
\text { familia }\end{array}$ & $\begin{array}{l}\text { Indicador compuesto para medir el grado } \\
\text { de satisfacción de las necesidades básicas } \\
\text { de las familias en un territorio, cuantifica } \\
\text { el grado de vulnerabilidad de la familia } \\
\text { en seis componentes: vulnerabilidad de la } \\
\text { familia, acceso al conocimiento, acceso al } \\
\text { empleo, disponibilidad de recursos econó- } \\
\text { micos, desarrollo infantil, condiciones de } \\
\text { la vivienda }\end{array}$ & (60) & \\
\hline $\begin{array}{l}\text { Índice de } \\
\text { vulnerabi- } \\
\text { lidad de la } \\
\text { salud }\end{array}$ & $\begin{array}{l}\text { Indicador compuesto que sintetiza } \\
\text { variables socioeconómicas y ambientales } \\
\text { consideradas esenciales para alcanzar la } \\
\text { ciudadanía: saneamiento, habituación, } \\
\text { educación, renta y sociales/salud }\end{array}$ & (61) & \\
\hline INIQUIS & $\begin{array}{l}\text { Es un indicador compuesto que usa un } \\
\text { conjunto de indicadores que dan cuenta } \\
\text { de las desigualdades entre poblaciones. } \\
\text { Este índice se encuentra constituido } \\
\text { por: esperanza de vida al nacer, tasa de } \\
\text { mortalidad de menores de un año, tasa } \\
\text { de mortalidad de menores de cinco años, } \\
\text { razón de mortalidad materna, partos } \\
\text { atendidos por personal de salud cualifica- } \\
\text { do, cobertura de la inmunización frente } \\
\text { al sarampión en niños menores de un } \\
\text { año, población que usa fuentes de agua } \\
\text { potable, número de médicos, número } \\
\text { de camas hospitalarias, gasto en salud } \\
\text { (porcentaje del PIB), gasto total en salud } \\
\text { per cápita al tipo de cambio promedio, } \\
\text { tasa global de fecundidad, PIB per cápita, } \\
\text { índice de concentración Gini, tasa de anal- } \\
\text { fabetismo en personas mayores de quince } \\
\text { años, porcentaje de personas pobres }\end{array}$ & $(22),(7)$ & \\
\hline
\end{tabular}




\begin{tabular}{|c|c|c|}
\hline $\begin{array}{l}\text { Índice de } \\
\text { Carencia } \\
\text { Social }\end{array}$ & $\begin{array}{l}\text { Proporción de viviendas sin recolección } \\
\text { de basura, sin suministro de agua potable, } \\
\text { sin alcantarillado, proporción de hogares } \\
\text { con cabeza de familia sin ingreso mensual } \\
\text { y proporción de hogares con cabeza de } \\
\text { familia sin educación }\end{array}$ & (62) \\
\hline $\begin{array}{l}\text { Índice de } \\
\text { privación } \\
\text { de los } \\
\text { hogares } \\
\text { (IPH) }\end{array}$ & $\begin{array}{l}\text { Hace distinción entre hogares con } \\
\text { privación y sin ella, reconoce tres formas } \\
\text { de privación: insuficiente capacidad eco- } \\
\text { nómica (privación de recursos corrientes), } \\
\text { condiciones habitacionales (privación } \\
\text { patrimonial) y privación convergente, que } \\
\text { es la sumatoria de las dos anteriores }\end{array}$ & (28) \\
\hline IDESE & $\begin{array}{l}\text { Índice sintético que abarca un conjunto de } \\
\text { doce indicadores sociales clasificados en } \\
\text { cuatro bloques: educación, renta, condi- } \\
\text { ciones de saneamiento y salud }\end{array}$ & (35) \\
\hline NBI & $\begin{array}{l}\text { Índice para medición de pobreza que in- } \\
\text { cluye calidad de la vivienda, hacinamiento, } \\
\text { inasistencia escolar y servicios públicos }\end{array}$ & (63) \\
\hline
\end{tabular}

Fuente: elaboración propia

Factores de riesgo. Finalmente, y pese a que no se puede decir que el enfoque de riesgo es semejante al de determinantes, es claro que en la región la medición de factores de riesgo también es usada para la evaluación de desigualdades en salud. Se encontró que dos estudios trabajaron estos indicadores desde diferentes contextos, ellos fueron: factores de riesgo para la mortalidad materna (23) y factores de riesgo para enfermedades respiratorias agudas en menores de cinco años (64). Estos artículos no discuten teóricamente la relación entre factores de riesgo y desigualdades en salud.

\subsection{Indicadores de resultado en salud. Los} indicadores de resultados más usados en las investigaciones sobre desigualdades en salud se muestran en la tabla 5, así como las investigaciones que los utilizaron.

TABla 5. IndiCADORES DE RESUltado EN SALUd USADOS DENTRO DE LAS INVESTIGACIONES SOBRE MORTALIDAD 2007-2014

\begin{tabular}{|l|l|l|l|}
\hline \multicolumn{1}{|c|}{$\begin{array}{c}\text { Tipo de } \\
\text { indicador }\end{array}$} & \multicolumn{1}{c|}{ Definición } & $\begin{array}{c}\text { Investigaciones } \\
\text { que lo usan }\end{array}$ & \multicolumn{1}{c|}{ Cometarios } \\
\hline $\begin{array}{l}\text { Número de muertes por mor- } \\
\text { talidad por cáncer de mama/ } \\
\text { cien mil mujeres mayores de } \\
\text { cuarenta años. } \\
\text { potal de defunciones por } \\
\text { por cáncer }\end{array}$ & $\begin{array}{l}\text { cáncer de boca ajustadas por } \\
\text { edad, raza yénero/ cien mil } \\
\text { habitantes. } \\
\text { Total de defunciones por cán- } \\
\text { cer de cuello uterino por grupo } \\
\text { de edad / población estimada } \\
\text { a mitad de periodo por cada } \\
\text { grupo de edad x 5 }\end{array}$ & $\begin{array}{l}\text { Este indicador fue } \\
\text { usado para cada } \\
\text { tipo de cáncer en } \\
\text { específico }\end{array}$ \\
\hline
\end{tabular}




\begin{tabular}{|c|c|c|c|}
\hline $\begin{array}{l}\text { Tasa de mortalidad } \\
\text { general }\end{array}$ & $\begin{array}{l}\text { Tasas de mortalidad por edad, } \\
\text { sexo y causa ajustadas por } \\
\text { edad mediante estandariza- } \\
\text { ción directa, tomando como } \\
\text { referencia la estructura etárea } \\
\text { de la población de Colombia } \\
\text { en el año } 2000 \\
\text { Tasa de mortalidad por estrato } \\
\text { por grupo de edad / cien mil } \\
\text { habitantes }\end{array}$ & $(46),(68)$ & \\
\hline $\begin{array}{l}\text { Tasa de mortalidad } \\
\text { infantil }\end{array}$ & $\begin{array}{l}\text { Número de niños muertos } \\
\text { menores de un año / número } \\
\text { de nacidos vivos }\end{array}$ & $\begin{array}{l}(32),(64),(60), \\
(57),(69),(15), \\
(17),(21),(22) \\
(25),(26),(70), \\
(71)(63),(72)\end{array}$ & $\begin{array}{l}\text { También se usó la } \\
\text { tasa de mortalidad } \\
\text { neonatal, que son las } \\
\text { muertes de niños an- } \\
\text { tes de los veintiocho } \\
\text { días de nacidos }\end{array}$ \\
\hline $\begin{array}{l}\text { Razón de mortalidad } \\
\text { por enfermedad } \\
\text { cardiovascular }\end{array}$ & $\begin{array}{l}\text { Relación de muertes ocurridas } \\
\text { en un periodo / muertes espe- } \\
\text { radas en el mismo periodo }\end{array}$ & (56), & \\
\hline $\begin{array}{l}\text { Tasa de mortalidad } \\
\text { por muertes violentas }\end{array}$ & $\begin{array}{l}\text { Suma de muertes por homi- } \\
\text { cidio, suicidio y accidentes de } \\
\text { tránsito }\end{array}$ & $\begin{array}{c}(20),(58),(73), \\
(59)\end{array}$ & \\
\hline $\begin{array}{l}\text { Esperanza de vida al } \\
\text { nacer }\end{array}$ & $\begin{array}{l}\text { Es el número medio de años } \\
\text { adicionales que una persona } \\
\text { podría vivir si las tendencias de } \\
\text { mortalidad al momento de su } \\
\text { nacimiento no cambian }\end{array}$ & $\begin{array}{l}(47),(52),(74), \\
(63)\end{array}$ & \\
\hline $\begin{array}{l}\text { Medición del fraccio- } \\
\text { namiento étnico }\end{array}$ & $\begin{array}{l}\text { Asociación entre la des- } \\
\text { igualdad de ingresos y los } \\
\text { indicadores de salud debido } \\
\text { a heterogeneidad étnica, va } \\
\text { de } 0 \text { a } 1\end{array}$ & (47) & \\
\hline $\begin{array}{l}\text { Años de vida poten- } \\
\text { cial perdida (AVPP) }\end{array}$ & $\begin{array}{l}\text { Relación entre la esperanza de } \\
\text { vida al nacer en el referente y } \\
\text { la esperanza de vida al nacer } \\
\text { del país de estudio }\end{array}$ & $\begin{array}{l}(75),(52),(76), \\
(77)\end{array}$ & \\
\hline $\begin{array}{l}\text { Razón de mortalidad } \\
\text { materna }\end{array}$ & $\begin{array}{l}\text { Número de mujeres que falle- } \\
\text { cen en un periodo determina- } \\
\text { do a causa de complicaciones } \\
\text { del embarazo / nacidos vivos } \\
\text { en ese mismo periodo }\end{array}$ & $(31),(73),(70)$ & \\
\hline $\begin{array}{l}\text { Tasa de mortalidad } \\
\text { por enfermedades } \\
\text { crónicas }\end{array}$ & $\begin{array}{l}\text { Incluido cáncer, enfermedades } \\
\text { respiratorias crónicas, diabe- } \\
\text { tes, enfermedades cerebrovas- } \\
\text { culares }\end{array}$ & (29) & \\
\hline Razón de tasas & $\begin{array}{l}\text { Se usa para el análisis de la } \\
\text { desigualdad entre dos grupos } \\
\text { de estudio }\end{array}$ & $\begin{array}{c}(77),(22),(25), \\
(26)\end{array}$ & \\
\hline $\begin{array}{l}\text { Sobremortalidad } \\
\text { (exceso de muertes) }\end{array}$ & $\begin{array}{l}\text { Diferencia entre las muertes } \\
\text { observadas y las esperadas }\end{array}$ & (17) & \\
\hline $\begin{array}{l}\text { Tasa de mortalidad } \\
\text { perinatal }\end{array}$ & $\begin{array}{l}\text { Número de muertes fetales } \\
\text { luego de las ocho semanas de } \\
\text { embarazo / mil nacimientos }\end{array}$ & (61) & \\
\hline
\end{tabular}




\begin{tabular}{|c|c|c|}
\hline $\begin{array}{l}\text { Coeficiente de mor- } \\
\text { talidad infantil por } \\
\text { causas evitables }\end{array}$ & $\begin{array}{l}\text { Muertes evitables por inmu- } \\
\text { noprevención, control del } \\
\text { embarazo, adecuada atención } \\
\text { del parto, diagnóstico y trata- } \\
\text { miento precoz }\end{array}$ & (48) \\
\hline $\begin{array}{l}\text { Tasa de mortalidad } \\
\text { por enfermedad } \\
\text { cerebrovascular }\end{array}$ & $\begin{array}{l}\text { Total de muertes por enfer- } \\
\text { medad cerebrovascular en } \\
\text { mayores } 45 \text { años/ total de la po- } \\
\text { blación residente mayor de } 45 \\
\text { años en un periodo de tiempo }\end{array}$ & (78) \\
\hline $\begin{array}{l}\text { Coeficiente de mor- } \\
\text { talidad por enferme- } \\
\text { dades del aparato } \\
\text { circulatorio }\end{array}$ & $\begin{array}{l}\text { Abarcó enfermedades } \\
\text { isquémicas del corazón, } \\
\text { enfermedades hipertensivas y } \\
\text { enfermedades cerebrovascula- } \\
\text { res / total de la población en el } \\
\text { periodo de estudio }\end{array}$ & $(35),(36)$ \\
\hline $\begin{array}{l}\text { Tasa de mortalidad } \\
\text { por diabetes mellitus }\end{array}$ & $\begin{array}{l}\text { Total de muertes por diabetes/ } \\
\text { total de la población en el } \\
\text { periodo estudiado }\end{array}$ & (37) \\
\hline $\begin{array}{l}\text { Coeficiente de mor- } \\
\text { talidad por raza }\end{array}$ & $\begin{array}{l}\text { Diferencia en mortalidad entre } \\
\text { blancos y negros en el periodo } \\
\text { de estudio }\end{array}$ & (79) \\
\hline $\begin{array}{l}\text { Tasa de fecundidad } \\
\text { global (TGF) }\end{array}$ & & $(63),(72)$ \\
\hline $\begin{array}{l}\text { Índice de transición } \\
\text { demográfica general }\end{array}$ & $\begin{array}{l}\text { Comparación entre la tasa de } \\
\text { mortalidad infantil y la tasa de } \\
\text { global de fecundidad }\end{array}$ & $(63),(72)$ \\
\hline $\begin{array}{l}\text { Incidencia acumu- } \\
\text { lada de mortalidad } \\
\text { infantil }\end{array}$ & $\begin{array}{l}\text { Número de muertes de niños } \\
\text { de uno a cuatro años x mil } \\
\text { niños que completaron en } \\
\text { primer año de vida }\end{array}$ & (32) \\
\hline
\end{tabular}

Fuente: elaboración propia

\section{Sobre la medición y el monitoreo de las desigualdades/inequidades en salud}

Alazraqui et al. (65) realizan una crítica a la epidemiología clínica y la medicina basada en la evidencia, puesto que estas se enfocan en el ámbito de la atención individual y descuidan el carácter poblacional que ha tenido la epidemiología. Plantean, entonces, que la epidemiología recobra su utilidad para la medición de las desigualdades en salud con la utilización de métodos descriptivos, ecológicos, de análisis multinivel y georreferenciales, siempre utilizando combinación de metodología cuantitativa y cualitativa que permita realizar triangulación.
Por su parte, Bacallao (80) presenta la polémica que hay acerca de la medición de las desigualdades en salud en la población. En general, existen dos posiciones, una que sostiene que las desigualdades deben ser medidas tomando como referencia toda la población, y la segunda, que la medición debe ser entre grupos socioeconómicos específicos. El autor se decide por la segunda opción, puesto que es más específica a la hora de estudiar características de los grupos sociales y se presta para menos sesgos. De igual manera, propone la utilización de indicadores basados en la noción de entropía, la cual define como una medida de desorden donde la salud y el ingreso están distribuidos 
de manera equitativa en una población; además, toma como referencia los indicadores de Theil y Kullback-Liebler (80).

En concreto, las medidas más usadas en Latinoamérica para el cálculo de la magni- tud de las desigualdades sociales en salud, a través del tiempo, incluyen la medición entre grupos sociales y entre individuos. Estas se presentan en la tabla 6 , sin hacer énfasis en la discusión metodológica de las implicaciones de cada uno de estos enfoques de medición.

Tabla 6. Medidas de Desigualdad usadas dentro de las inVestigaciones SOBRE MORTALIDAD Y AUTOPERCEPCIÓN EN SALUD 2007-2014

\begin{tabular}{|c|c|c|}
\hline Tipo de indicador & Definición & $\begin{array}{l}\text { Investigaciones que } \\
\text { lo usan }\end{array}$ \\
\hline $\begin{array}{l}\text { Índice Foster-Grer-Thor- } \\
\text { becke (FGT) }\end{array}$ & $\begin{array}{l}\text { Se basa en la estimación de la distancia } \\
\text { entre una persona pobre y una rica, } \\
\text { déficit de ingresos }\end{array}$ & (57) \\
\hline $\begin{array}{l}\text { Índice Sen-Shorroscks-Sen } \\
\text { (SST) }\end{array}$ & $\begin{array}{l}\text { Proporción de gente pobre, el nivel } \\
\text { de la pobreza y la distribución del } \\
\text { bienestar }\end{array}$ & (57) \\
\hline $\begin{array}{l}\text { Riesgo atribuible poblacio- } \\
\text { nal (RAP) }\end{array}$ & $\begin{array}{l}\text { Permite medir el impacto total de la } \\
\text { desventaja social sobre algún evento, } \\
\text { tomando en cuenta el tamaño de los } \\
\text { diferentes grupos sociales }\end{array}$ & (21) \\
\hline Fracción atribuible (FA) & $\begin{array}{l}\text { Representa el riesgo que se tiene a } \\
\text { morir por vivir en el país del estudio } \\
\text { y no en el país que se escogió como } \\
\text { referente internacional }\end{array}$ & $(24),(36)$ \\
\hline $\begin{array}{l}\text { Índice de mortalidad } \\
\text { infantil }\end{array}$ & $\begin{array}{l}\text { Defunciones infantiles observadas / } \\
\text { defunciones infantiles esperadas }\end{array}$ & (27) \\
\hline Distancia de GOWER & $\begin{array}{l}\text { Indicador sintético construido a partir } \\
\text { de dos indicadores, mide disparidades } \\
\text { en salud y socioeconómica }\end{array}$ & (27) \\
\hline Índice de desigualdad & $\begin{array}{l}\text { Se basa en el nivel educativo, se } \\
\text { construye de mayor a menor desde } 0 \\
\text { a } 100 \% \text {, de esta manera se interpreta } \\
\text { la proporción de mortalidad entre } \\
\text { personas con nivel de educación o } \\
\text { comparado con el grupo de nivel edu- } \\
\text { cativo } 100 \%\end{array}$ & (34) \\
\hline $\begin{array}{l}\text { Riesgo relativo de mortali- } \\
\text { dad por nivel educativo }\end{array}$ & $\begin{array}{l}\text { Proporción de mortalidad entre perso- } \\
\text { nas con nivel } 0 \text { de educación y personas } \\
\text { con nivel } 100 \% \text { de educación }\end{array}$ & (34) \\
\hline $\begin{array}{l}\text { Índice de desigualdad } \\
\text { dependiente, Îndice relativo } \\
\text { de desigualdad }\end{array}$ & $\begin{array}{l}\text { Se requiere crear una variable inter- } \\
\text { mediaria que sería un indicador de la } \\
\text { posición de la mortalidad en relación } \\
\text { con el nivel socioeconómico }\end{array}$ & (36) \\
\hline $\begin{array}{l}\text { Coeficiente Gini en la dis- } \\
\text { tribución de ingresos }\end{array}$ & $\begin{array}{l}\text { Indicador que expresa la desigualdad } \\
\text { en la distribución de renta }\end{array}$ & (46), (47), (48) \\
\hline Índice de concentración & $\begin{array}{l}\text { Muertes infantiles, escolaridad mater- } \\
\text { na y renta domiciliaria per cápita }\end{array}$ & (50) \\
\hline
\end{tabular}

26 Fuente: elaboración propia 


\section{Discusión}

Se encontró que el país con mayor proporción de producción investigativa en el tema es Brasil, lo que constituye una tendencia que se mantiene en el tiempo y que se ha verificado en anteriores revisiones (8-10). En contraste con lo hallado por otros estudios: $36.4 \%$ de Almeida-Filho en 1999 y 2003 $(8,9), 54.2 \%$ de Raiher en el 2008 (10) y $85.7 \%$ de Muntaner et al. en el 2012 (13). Se debe aclarar que, en este último caso, la revisión estuvo enfocada en el tema de clase social como determinante de la salud. Lo anterior puede tener una relación con el tamaño del país y sus instituciones, sumado a la gran fuerza del movimiento de la salud colectiva brasileña, la promulgación de la salud como derecho fundamental en la Constitución Política de 1988 y a la instauración del Sistema Unico de Saude (SUS), referente de los sistemas de salud universales en la región latinoamericana, sumado al hecho de las grandes desigualdades socioeconómicas que han caracterizado a la sociedad brasilera, lo que probablemente ha generado interés por parte de investigadores y expertos acerca del tema.

Dentro de los países que han hecho aportes a la investigación sobre el tema se encuentra Chile, que en los estudios de Almeida $(8,9)$ tuvo una producción de $7.4 \%$, mientras que en nuestros resultados es del $4 \%$, lo que muestra una disminución en la producción regional de estos trabajos. Para Argentina se mantiene la proporción del total de publicaciones, en tanto que en los estudios de Almeida $(8,9)$ la producción fue de $4.8 \%$, mientras que nuestros hallazgos esa proporción es de $5 \%$ sobre el total.

Cabe destacar que en los estudios de Almeida la mayor parte de la producción se concentra, de mayor a menor, en Brasil, México, Chile y Argentina, con cerca de un $70 \%$ de las publicaciones, seguidos de Ecuador y Cuba $(8,9)$. En el caso de los cuatro primeros es bastante llamativo, por ser las primeras cuatro economías de la región latinoamericana, lo que podría traducirse en mayores recursos económicos y apoyo a los procesos investigativos. Esta tendencia cambia en nuestros hallazgos, en los cuales se observa un aumento considerable de publicaciones en el caso de Colombia y Cuba, desplazando a Chile y Argentina como países con mayor proporción de publicaciones en el tema. Si se toma como referencia los trabajos del mencionado autor $(8,9)$ y se contrastan sus resultados con los nuestros, en el caso de Colombia se pasa de menos del $2 \%$ al $20 \%$ y en el caso de Cuba se pasa de 2.6 a $7 \%$ de publicaciones. En cuanto a México, sigue siendo un país que se mantiene entre los que más producción genera sobre el tema, con un porcentaje que pasa de 8.6 a $12 \%, 1.4$ veces más. Lo anterior puede deberse a varios fenómenos, dentro de los que se cuentan la crisis del sistema de salud colombiano de los últimos años, con el empeoramiento de los indicadores de salud para la mayoría de la población; las reformas aplicadas al sistema de salud mexicano con la implementación del denominado seguro popular para la población más pobre, y una transición sociopolítica en Cuba, que ha llevado a que las condiciones de vida de la población no parezcan ser tan homogéneas como antes. Todo lo anterior, definitivamente, pudo haber despertado mayor interés investigativo sobre el tema de las desigualdades/inequidades en salud en estos tres países. Por otra parte, llama la atención que en nuestra revisión no se encontraron estudios realizados en Ecuador, contrariamente a los trabajos de Almeida $(8,9)$. Esto es bastante sorprendente, si se tiene en cuenta que Ecuador ha sido un país que ha realizado grandes aportes a la discusión y producción científica en los movimientos de la medicina social latinoamericana y la epidemiología crítica. 
En el ámbito de los principales centros de producción, hay una fuerte concentración en las instituciones brasileñas y colombianas. En el caso de las instituciones brasileñas, la Fundación Oswaldo Cruz se encuentra a la cabeza, lo que concuerda con los estudios de Raiher (10) y Almeida $(8,9)$. También existe bastante similitud respecto a las revistas donde se publicaron los artículos de investigación. En nuestro estudio las revistas donde más se encontraron publicaciones fueron Cadernos de Saude Publica, Revista Facultad Nacional de Salud Pública, Revista de Salud Pública, Ciencia y Saude Coletiva, Revista Panamericana de Salud Pública, Revista de Saude Pública, Revista Cubana de Salud Pública y Revista Brasilera de Epidemiologia. Lo anterior concuerda con la revisión de Raiher, en la cual las revistas con mayores publicaciones fueron Cadernos de Saude Publica, Revista de Salud Pública, Revista de Saude Publica, Ciencia y Saude Coletiva y Revista Brasilera de Epidemiologia. Esto refuerza el impacto que tiene Brasil, no solo en la producción sobre el tema, sino sobre sobre la publicación, puesto que Fiocruz y Abrasco, como instituciones representativas, demuestran ser medios importantes para la difusión.

En el presente trabajo las principales metodologías empleadas son los estudios teóricos, algo similar a los resultados de Almeida $(8,9)$. En cuanto a los temas, en nuestro estudio priman los debates teóricos/metodológicos, la mortalidad maternoinfantil y la mortalidad por enfermedades crónicas.

Se identificaron cuatro categorías emergentes respecto a los determinantes de las inequidades: condiciones socioeconómicas de individuos y familias, género, etnia/raza y condiciones socioeconómicas de unidades territoriales. En condiciones de clase predomina el enfoque neomarxista, análogamente a los hallazgos de Almeida y Muntaner
$(8,9,13)$. Respecto a etnia/raza y género no se presenta una discusión profunda acerca de estos determinantes, como en los resultados de Almeida (8,9). En el caso de la medición de inequidades por unidades geográficas no hay una discusión teórica sobre la categoría territorio que realice una reflexión sobre el tema.

\section{A manera de conclusión}

Esta investigación demuestra que hay interés creciente en la región por estudiar las desigualdades/inequidades en salud, por lo menos en el campo de la mortalidad; sin embargo, no siempre existe articulación entre los estudios empíricos y los debates teórico-metodológicos sobre el tema. En general, pareciera que las discusiones teóricas se generan en unos lugares y las investigaciones empíricas en otros. Se encuentra asî una brecha entre la discusión teórico-metodológica y la investigación empírica, sobre la cual es necesario profundizar para poder comprenderla.

Asimismo, se encontró que la producción académica en la región es muy desigual, Brasil es el país que genera la mayor proporción de dicha producción, lo que se constituye en una tendencia que se mantiene en el tiempo y que se ha verificado en anteriores revisiones.

En cuanto a las redes de colaboración, se encontró que la gran mayoría se presenta entre instituciones brasileñas, con muy poco trabajo en red entre universidades latinoamericanas; también se hallaron trabajos de investigación en red entre universidades de Latinoamérica y algunas universidades norteamericanas y una red que involucra instituciones de Norteamérica, Suramérica y Europa. Dentro de las redes de colaboración se logró identificar una que trabaja de forma repetida: Universidad Nacional de Lanús 
con la Universidad Federal de Bahía. Sin embargo, hay que reconocer que predomina una producción endógena, y por ello es necesario asumir como reto regional el trabajo colaborativo sur-sur y sur-norte.

En los estudios que abordan los determinantes de las desigualdades sociales en salud, la mayoría adopta el modelo de determinantes sociales de la salud de la OMS; seguramente, la ausencia de estudios empíricos desde otros enfoques, como el ecosocial y la determinación social, obedece a las dificultades que aún puede haber en su difusión, conocimiento y operacionalización. Aquí hay un reto teórico-metodológico con respecto a explorar opciones de complementariedad hasta donde sea posible y de acuerdo a los diferentes paradigmas que sustentan los modelos teóricos.

Una de las grandes dificultades planteadas por diversos autores para el estudio de las desigualdades/inequidades en salud en la región es el subregistro de información y la calidad de los datos, principalmente en las estadísticas vitales como mortalidad, esperanza de vida al nacer y años de vida potencial perdidos, así como la falta de registros sistemáticos que tengan en cuenta categorías como clase, etnia y género, e incluso la existencia de información socioeconómica de unidades territoriales que pueda ser útil para el análisis de las desigualdades, entre otras $(51,75,76)$. Seguramente, los problemas de cobertura y calidad de los datos es otro de los elementos que explicarían la dificultad para operacionalizar abordajes teóricos diferentes en la región.

Finalmente, y sobre esto se debe llamar la atención, la discusión sobre inequidades en salud se inscribe en los debates de justicia social. Estos debates están presentes en la región, pero aún planteados en el campo teórico e independientes de estudios empíricos, lo cual genera una brecha adicional entre la discusión de justicia sanitaria y las desigualdades en salud. Usualmente, cuando se plantea la inequidad en las investigaciones empíricas, se acoge la perspectiva de Whitehead, pese a lo cual el asunto no lo plantean explícitamente todas las investigaciones.

En síntesis, se puede decir que, a pesar de la importancia del tema para la investigación en Latinoamérica, hay retos muy importantes, como la articulación más estrecha entre la discusión teórico-metodológica sobre justicia social y los determinantes de las desigualdades en salud y los estudios empíricos, y esto implica una reflexión en dos sentidos: plantear de manera explícita las perspectivas teóricas y sus implicaciones en la investigación empírica y discutir — desde la investigación empírica- los aportes teóricos. Asimismo, es necesario avanzar en procesos de colaboración sur-sur entre los mismos países de Latinoamérica, para fortalecer la investigación en el conjunto de la región y así ampliar la comprensión sobre las desigualdades en la región y las formas concretas que la explican. En este segundo punto los estudios epidemiológicos deberían ser complementados con estudios cualitativos que permitan ampliar el horizonte de comprensión.

\section{Limitaciones del estudio}

Este trabajo no puede generalizarse, ya que es una revisión sistemática de dos bases de datos indexadas en la región, y no incluyó producción académica diferente como libros, capítulos de libro o trabajos de investigación de posgrado. Es importante aclararlo, porque es posible que el estudio de las desigualdades/inequidades desde otros modelos teóricos se esté desarrollando y circulando en otros escenarios que no sean las publicaciones indexadas en estas dos bases de datos - Scielo y Lilacs-. Esta revisión 
aportaría a construir un estado del arte de la investigación sobre el tema en la región, pero no constituye un estado del arte.

\section{Referencias bibliográficas}

1. Whitehead M, Dahlgren G, Evans T. Equity and health sector reforms: can low-income countries escape the medical poverty trap? The Lancet. 2001;358(9284):833-6.

2. Muntaner C, Benach J, Tarafa G, Chung H. The welfare state and global health: Latin America, the Arab world and the politics of social class. Gac Sanit. 2011;25(6):445-7.

3. Uribe M. Los vaivenes de las políticas sociales en Argentina, Colombia, Chile, México y Uruguay ¿Neo o postneoliberalismo? Guanajuato: Editorial Porrúa; 2011.

4. Jiménez JP. Desigualdad, concentración del ingreso y tributación sobre las altas rentas en América Latina. Santiago de Chile: Comisión Económica para América Latina y el Caribe (Cepal); 2015.

5. Ortiz I, Cummins M. Desigualdad global: La distribución del ingreso en 141 países. Documento de trabajo sobre política económica y social. Nueva York: Unicef; 2014.

6. Benach J, Vergara M, Muntaner C. Desigualdad en salud: la mayor epidemia del siglo XXI. Papeles Relac Ecosociales Cambio Glob. 2008;(103):29-40.

7. Cardona D, Acosta LD, Bertone CL. Inequidades en salud entre países de Latinoamérica y el Caribe (2005-2010). Gac Sanit 2013 jul.;27(4):292-7.

8. Almeida-Filho N. Desigualdades em saúde segundo condições de vida: análise da produção científica na América Latina e Caribe e bibliografia anotada. En: OPAS Pesquisa em Saúde Pública Documentos Técnicos [internet]. Organizaçao Pan-americana da Saúde; 1999 [acceso: 27 de julio del 2016]. Disponible en: http://bases.bireme.br/cgi-bin/wxislind.exe/ iah/online/?IsisScript $=$ iah/iah.xis\&src $=$ google \&base $=$ LILACS\&lang $=$ p\&nextAction $=$ lnk\&exprSearch $=380521 \&$ indexSearch $=$ ID

9. Almeida-Filho N, Kawachi I, Filho AP, Dachs JNW. Research on health inequalities in Latin America and the Caribbean: bibliometric analysis (19712000) and descriptive content analysis (1971-1995). Am J Public Health. 2003;93(12):2037-43.

10. Raiher S. Estudio comparativo entre la producción científica latinoamericana y mundial en inequidades en salud durante los años 1999-2008. [Trabajo final de grado de Licenciada en Bibliotecología y Documentación]. La Plata: Universidad Nacional de La Plata, Facultad de Humanidades y Ciencias de la Educación; 2010.

11. Dos Santos D, Gerhardt T. Desigualdades sociais e saúde no Brasil: produção científica no contexto do Sistema Único de Saúde. Rev Gaúcha Enferm. 2008;2(3):129-36.

30 12. Borde E, Akerman M, Pellegrini Filho A. Mapping of capacities for research on health and its social determinants in Brazil. Cad Saúde Pública. 2014;30(10):2081-91.

13. Muntaner C, Rocha KB, Borrell C, Vallebuona C, Ibáñez C, Benach J, et al. Social class and health in Latin America. Rev Panam Salud Pública. 2012;31(2):166-75.

14. Álvarez Pérez A, Gonzálvez L, Pilar I, Salvia R, Jorge A, Gorbea B, et al. Actualización conceptual sobre los determinantes de la salud desde la perspectiva cubana. Rev Cuba Hig Epidemiol. 2010;48(2).

15. Medina-Gómez OS, López-Arellano O. Asociación de los tipos de carencia y grado de desarrollo humano con la mortalidad infantil en México, 2008. Cad Saúde Pública. 2011;27(8):1603-10.

16. Turci SRB, Figueiredo VC, de Azevedo Lozana J. Auto-avaliação de saúde e limitações físicas decorrentes de problemas de saúde. Rev Saúde Pública. 2008;42(4):741-9.

17. Murakami GF, Guimarães MJB, Sarinho SW. Desigualdades sociodemográficas e causas de morte em menores de cinco anos no Estado de Pernambuco. Rev Bras Saúde Matern Infant. 2011;11(2):139-52.

18. Fernández JCA. Determinantes culturais da saúde: uma abordagem para a promoção de equidade. Saúde E Soc. 2014;23(1):167-79.

19. Santos JAF. Desigualdade racial de saúde e contexto de classe no Brasil. DADOS-Revista Ciênc Sociais. 2011;54(1):5-40.

20. Araújo EM, Costa M da CN, Hogan VK, Araújo TM de, Batista A, Oliveira LOA. The use of the race/color variable in public health: possibilities and limitations. Interface. Comun Saúde Educ. 2010;5.

21. Flores F M, Cerda LJ. Evolución de la desigualdad en la mortalidad infantil. Rev Chil Pediatría. 2010;81(3):215-20.

22. González-Pérez GJ, Vega-López MG, Romero-Valle S, Vega-López A, Cabrera-Pivaral CE. Exclusión social e inequidad en salud en México: Un análisis socio-espacial. Rev Salud Pública. 2008;10:15-28.

23. Leite RMB, Araújo TVB, Albuquerque RM, Andrade ARS, Neto D, José P. Fatores de risco para mortalidade materna em área urbana do Nordeste do Brasil. Cad Saúde Pública. 2011;27(10):1977-85.

24. Sandoval-Vargas YG, Eslava-Schmalbach JH. Inequality regarding maternal mortality in Colombian departments in 2000-2001, 2005-2006 and 2008-2009. Rev Salud Pública. 2013;15(4):579-91.

25. Mosquera Méndez PA, Granados Hidalgo G, Vega Romero R. La estrategia de atención primaria en salud (APS) para Bogotá-Colombia y su relación con la disminución de inequidades de resultados en salud. Gerenc Políticas Salud [internet]. 2008 [acceso: 27 de julio de 2016];7(14). Disponible en: http://revistas.javeriana.edu.co/index.php/gerepolsal/article/view/2682

26. Augsburger AC, Gerlero SS, Galende S, Moyano CB. La expresión de las desigualdades sociales en la mortalidad infantil. Información epidemiológica en regiones seleccionadas de la provincia de Santa Fe (Argentina). Rev Fac Nac Salud Pública. 2013;31:139-48.

27. Chamizo García H, Behm Ammazzini I. Las inequidades geográficas en la mortalidad infantil en Costa Rica, período 2008-2012. Poblac Salud en Mesoamérica. 2014;12(1):1-20. 
28. Souza M de L de, Laurenti R, Knobel R, Monticelli M, Brüggemann OM, Drake E, et al. Mortalidad maternal en Brasil debida a hemorragia. Rev Lat Am Enfermagem. 2013 jun.21(3):711-8.

29. Stevens A, Schmidt MI, Duncan BB. Desigualdades de gênero na mortalidade por doenças crônicas não transmissíveis no Brasil. Ciênc Saúde Coletiva. 2012;17(10):2627-34.

30. Marchiori Buss P, Pellegrini Filho A. A saúde e seus determinantes sociais. Physis Rev Saúde Coletiva. 2007;17(1):77-93.

31. Lazcano-Ponce E, Schiavon R, Uribe-Zúñiga P, Walker D, Suárez-López L, Luna-Gordillo R, et al. Cobertura de atención del parto en México: Su interpretación en el contexto de la mortalidad materna. Salud Pública México. 2013;55(Supl 2):S214-24.

32. Horta BL, Gigante DP, Candiota JS, Barros FC, Victora CG. Monitorização da mortalidade na coorte de nascimentos de 1982 a 2006, Pelotas, RS. Rev Saúde Pública. 2008;42(suppl. 2):108-14.

33. Geib LTC. Determinantes sociais da saúde do idoso. Ciênc Amp Saúde Coletiva. 2012;17(1):123-33.

34. Arroyave I, Burdorf A, Cardona D, Avendano M. Socioeconomic inequalities in premature mortality in Colombia, 1998-2007: The double burden of non-communicable diseases and injuries. Prev Med. 2014;64:41-7.

35. Gotler Medeiros CR, Meneghel SN, Engel Gerhardt T. Desigualdades na mortalidade por doenças cardiovasculares em pequenos municipios. Ciênc Amp Saúde Coletiva. noviembre de 2012;17(11):2953-62.

36. Bassanesi SL, Azambuja MI, Achutti A. Mortalidade precoce por doenças cardiovasculares e desigualdades sociais em Porto Alegre: da evidência à ação. Arq Bras Cardiol. 2008;90(6):403-12.

37. Klafke A, Duncan BB, Rosa R dos S, Moura L de, Malta DC, Schmidt MI. Mortalidade por complicações agudas do diabetes melito no Brasil, 2006-2010. Epidemiol E Serviços Saúde. 2014;23(3):455-62.

38. Cabieses B, Rice M, Muñoz M, Zuzulich MS. Igualdad y equidad: pasos necesarios para construir una universidad más saludable. Rev Fac Nac Salud Pública. 2011;29(3):308-19.

39. Vieira-da-Silva LM, Filho A, De N. Eqüidade em saúde: uma análise crítica de conceitos. Cad Saúde Pública. 2009;25:s217-26.

40. Orozco AS. Justicia global: sus implicaciones para el manejo de las inequidades en salud. Rev Fac Nac Salud Pública. 2013;31(Supl 1):65-72.

41. Pineda Restrepo B del C. Desarrollo humano y desigualdades en salud en la población rural en Colombia. Univ Odontol. 2011;31(66):97-102.

42. Acero M, Caro IM, Henao L, Ruiz LF Sánchez G. Social determinants of health: official stance and critical views. Rev Fac Nac Salud Pública. 2013;31(Supl 1):103-10.

43. Franco-Giraldo A, Palma M, Álvarez-Dardet C. Efecto del ajuste estructural sobre la situación de salud en América Latina y el Caribe, 1980-2000. Rev Panam Salud Publica. 2006;19(5):291-9.
44. Barata RB, Ribeiro MCS de A, Silva ZP da, Antunes JLF, Barata RB, Ribeiro MCS de A, et al. Social class: concepts and operationalization models in health research. Rev Saúde Pública. 2013;47(4):647-55.

45. Antunes JLF. Condições socioeconômicas em saúde: discussão de dois paradigmas. Rev Saúde Pública. 2008;42(3):562-7.

46. Rodríguez García J. Desigualdades socioeconómicas entre departamentos y su asociación con indicadores de mortalidad en Colombia en 2000. Rev Panam Salud Pública. 2007;21(2-3):111-24.

47. Idrovo AJ, Ruiz-Rodríguez M, Manzano-Patiño AP. Beyond the income inequality hypothesis and human health: a worldwide exploration. Rev Saúde Pública. 2010;44(4):695-702.

48. Boing AF, Boing AC. Mortalidade infantil por causas evitáveis no Brasil: um estudo ecológico no período 2000-2002. Cad Saúde Pública. 2008;24(2):447-55.

49. Ventura RN, Puccini RF, Silva NN, Silva EMK, Oliveira EM. The expression of vulnerability through infant mortality in the municipality of Embu. Sao Paulo Med J. 2008;126(5):262-8.

50. Garcia LP, Santana LR. Evolução das desigualdades socioeconómicas namortalidade infantil no Brasil, 1993-2008. Ciênc Amp Saúde Coletiva. 2011;16(9):3717-28.

51. Artiles-Visbal L. El nudo gordiano de la equidad de género. Rev Salud Pública. diciembre de 2008;10:109-18.

52. Pedroso Zulueta T. Gender disparities in mortality: Challenges for health equity in Puerto Rico. Acta Colomb Psicol. 2013;16(2):103-14.

53. Denman CA, V C, Carmen M, Aranda P. Salud en Sonora desde una perspectiva de género: retos y propuestas. Región Soc. 2007;19:147-70.

54. Gonçalves AC, Costa MCN, Paim JS, Silva LMV, Braga JU, Barreto M, et al. Social inequalities in neonatal mortality and living condition. Rev Bras Epidemiol. septiembre de 2013;16(3):682-91.

55. Barros MB de A. Inquéritos domiciliares de saúde: potencialidades e desafios. Rev Bras Epidemiol. 2008;11(supl.1):6-19.

56. Nogueira MC, Ribeiro LC, Cruz OG. Desigualdades sociais na mortalidade cardiovascular precoce em um município de médio porte no Brasil. Cad Saúde Pública. 2009;25(11):2321-32.

57. Servan-Mori E, Torres-Pereda P, Orozco E, Sosa-Rubí SG. An explanatory analysis of economic and health inequality changes among Mexican indigenous people, 2000-2010. Int J Equity Health. 2014;13:21.

58. Viana LAC, Costa M da CN, Paim JS, Vieira-da-Silva $\mathrm{LM}$. Social inequalities and the rise in violent deaths in Salvador, Bahia State, Brazil: 2000-2006. Cad Saúde Pública. 2011;27:s298-308.

59. Ribeiro da Costa IE, Ludermir AB, Avelar Silva I. Diferenciais da mortalidade por violência contra adolescentes segundo estrato de condição de vida e raça/cor na cidade do Recife. Ciênc Amp Saúde Coletiva. 2009;14(5):1781-8. 
60. Ramalho WM, Sardinha LMV, Rodrigues IP, Duarte EC. Inequalities in infant mortality among municipalities in Brazil according to the Family Development Index, 2006-2008. Rev Panam Salud Pública Pan Am J Public Health. 2013;33(3):205-12.

61. Martins EF, Rezende EM, de Mattos Almeida MC, Lana FCF. Mortalidad perinatal y desigualdades socio-espaciales. Rev Latino-Am Enfermagem. 2013;21(5):1062-70.

62. Vilela MBR, Bonfim C, Medeiros Z. Mortalidade infantil por doenças infecciosas e parasitárias: reflexo das desigualdades sociais em um município do Nordeste do Brasil. Rev Bras Saúde Materno Infant. 2008;8(4):445-61.

63. Urrea Giraldo F, Sánchez R, Alejandro D. Transformaciones sociodemográficas y de condiciones de vida en dos pueblos indígenas y poblaciones negras en el norte del Cauca en el período intercensal 1993-2005. Colomb Médica. 2012;43(2):126-32.

64. Cardoso AM, Coimbra JCE, Barreto CTG, Werneck GL, Santos RV. Mortality among Guarani Indians in Southeastern and Southern Brazil. Cad Saúde Pública. 2011;27:s222-36.

65. Alazraqui M, Mota E, Spinelli H, Guevel C. Desigualdades en salud y desigualdades sociales: un abordaje epidemiológico en un municipio urbano de Argentina. Rev Panam Salud Pública. 2007;21(1):1-10.

66. Antunes JLF, Toporcov TN, Biazevic MGH, Boing AF, Bastos JL, Antunes JLF, et al. Gender and racial inequalities in trends of oral cancer mortality in Sao Paulo, Brazil. Rev Saúde Pública. 2013;47(3):470-8.

67. Martínez ML, Guevel CG. Desigualdades sociales en la mortalidad por cáncer de cuello de útero en la Ciudad Autónoma de Buenos Aires, 1999-2003 y 2004-2006. Salud Colect. 2013;9(2):169-82.

68. Cruz SA, Vieira-da-Silva LM, Costa M da CN, Paim JS. Evolution of inequalities in mortality in Salvador, Bahia State, Brazil, 1991/2006. Cad Saúde Pública. 2011;27:s176-84.

69. Frenz P, González C. Aplicación de una aproximación metodológica simple para el análisis de las desigualdades: El caso de la mortalidad infantil en Chile. Rev Médica Chile. 2010;138(9):1157-64.
70. Ortunio E. Tendencias y desigualdades territoriales de la mortalidad infantil y materna. estado Carabobo, periodo 1990-2007. Comunidad Salud. 2012;10(2):2-13.

71. Lansky S, Friche AAL, Silva AAM, Campos D, Bittencourt SDA, Carvalho ML, et al. Birth in Brazil survey: neonatal mortality, pregnancy and childbirth quality of care. Cad Saúde Pública. 2014;30:S192-207.

72. Grajales IC, Cardona AD. The second demographic transition and the development level of Colombia departments, 2005. Rev Fac Nac Salud Pública. 2010;28(3):209-20.

73. Souza MLP, Orellana JDY. Desigualdades na mortalidade por suicídio entre indígenas e não indígenas no estado do Amazonas, Brasil. J Bras Psiquiatr. 2013;62(4):245-52.

74. González-González C, Samper-Ternent R, Wong $\mathrm{R}$, Palloni A. Mortality inequality among older adults in Mexico: the combined role of infectious and chronic disease. Rev Panam Salud Pública Pan Am J Public Health. 2014;35(2):89-95.

75. Eslava-Schmalbach J, Rincón CJ, Guarnizo-Herreño CC. Inequidad en los años de vida potencial perdida, por departamentos en Colombia 19852005. Rev Salud Pública. 2012;13(1):1-12.

76. Eslava-Schmalbach JH, Rincón CJ, Guarnizo-Herreño CC. 'Inequidad' de la expectativa de vida al nacer por sexo y 'departamentos' de Colombia. Biomédica. 2013;33(3):383-92.

77. Dos Santos SM, Bezerra Guimarães MJ, Barreto de Araújo TV. Desigualdades raciais na mortalidade de mulheres adultas no Recife, 2001 a 2003. Saúde E Soc. 2007;16(2):87-102.

78. Furukawa T, Thais Aidar de Freitas M, Marcon SS, et al. Mortalidade por doenças cerebrovasculares por residência e local de ocorrência do óbito: Paraná, Brasil, 2007. Cad Saúde Pública. 2011;27(2):327-34.

79. Fiorio NM, Flor LS, Padilha M, Castro DS, Molina M del CB. Mortalidade por raça/cor: evidências de desigualdades sociais em Vitória (ES), Brasil. Rev Bras Epidemiol. 2011;14(3):522-30.

80. Bacallao Gallestey J. Indicadores basados en la noción de entropía para la medición de las desigualdades sociales en salud. Rev Cuba Salud Pública. 2007;33(4). 